\title{
Bootstrap Methods for a Measurement Estimation Problem
}

\author{
José I. De la Rosa, Member, IEEE, and Gilles A. Fleury
}

\begin{abstract}
In this paper, a new approach for the statistical characterization of a measurand is presented. A description of how different bootstrap techniques can be applied in practice to estimate successfully a measurand probability density function (pdf) is given. When the direct observation of a quantity of interest is practically impossible such as in nondestructive testing, it is necessary to estimate such quantity, which is also called measurand. The statistical characterization of any estimator is important, because all the uncertainty features can be accessible to qualify such estimator. On the other hand, most of the time, the large-scale repetition of an experiment is not economically feasible, so that the Monte Carlo methods cannot be used directly for uncertainty characterization. Bootstrap methods have proved to be a potentially useful alternative. Moreover, a biased bootstrap recent technique, with which robust parameter estimates are obtained, is used. This technique is extended to be used in measurand estimation. An extended nested bootstrap improvement for the measurand pdf estimation is also presented. These techniques are applied to a realistic multidimensional measurand-estimation problem of groove dimensioning using remote field eddy current inspection. Measurand uncertainty characterization using the bootstrap techniques generally gives an accurate pdf estimation.
\end{abstract}

Index Terms-Bootstrap, indirect measurement, Monte Carlo simulation, nonlinear regression, probability density function (pdf) estimation.

\section{NOTATIONS AND ACRONYMS}

\begin{tabular}{|c|c|}
\hline$a, \mathbf{a}, \mathbf{A}$ & Scalar, vector, and matrix. \\
\hline $\mathbf{a}^{\top}, \mathbf{A}^{\top}$ & Transposed vector and matrix. \\
\hline$y_{i}, \mathbf{y}$ & Vector of observations (data). \\
\hline$x_{i}, \boldsymbol{x}$ & $\begin{array}{l}\text { Experimental protocol or instrumental para- } \\
\text { meters. }\end{array}$ \\
\hline$f(\boldsymbol{x}, \boldsymbol{\theta}), f(\cdot)$ & Model function parameterized by $\boldsymbol{x}$ and $\boldsymbol{\theta}$. \\
\hline$\theta$ & Unknown parameter vector. \\
\hline$g(\boldsymbol{\theta}), g(\cdot)$ & $\begin{array}{l}\text { Measurand and parameter functional relation- } \\
\text { ship. }\end{array}$ \\
\hline$G_{k}(\boldsymbol{\theta}), G_{k}(\cdot)$ & $\begin{array}{l}\text { Bijective relation between measurand and pa- } \\
\text { rameters. }\end{array}$ \\
\hline$\wp(\boldsymbol{\theta})$ & Parameter distribution. \\
\hline$\wp(m), \wp(\boldsymbol{m})$ & Measurand distribution. \\
\hline$\wp(e)$ & Errors or noise distribution. \\
\hline$\widehat{\boldsymbol{\theta}}, \bar{\theta}$ & Parameter estimator and real parameters. \\
\hline$p$ & Dimensions of vector $\boldsymbol{\theta}$. \\
\hline
\end{tabular}

Manuscript received May 3, 2005; revised October 7, 2005.

J. I. De la Rosa is with the Signal Processing Laboratory, Engineering Faculty, Universidad Autónoma de Zacatecas, Zacatecas, Mexico (e-mail: ismaelrv@ieee.org).

G. A. Fleury is with the École Supérieure d'Électricité-Department of Signal Processing and Electronic Systems, Plateau de Moulon, Gif-sur-Yvette Cedex 91192, France (e-mail: Gilles.Fleury@ supelec.fr).

Digital Object Identifier 10.1109/TIM.2006.873779

$\begin{array}{ll}n & \text { Dimensions of vectors } \boldsymbol{y}, \boldsymbol{x}, \text { and } \boldsymbol{e} . \\ \widehat{e}_{i}, \widehat{\boldsymbol{e}} & \text { Residuals vector. } \\ \widehat{\wp}_{e}, \widehat{\wp}(\boldsymbol{e}) & \text { Residuals empiric distribution. } \\ e_{i}^{*}, \boldsymbol{e}^{*} & \text { Bootstrap residuals vector. } \\ y_{i}^{*}, \mathbf{y}^{*} & \text { Bootstrap fictive data. } \\ \widehat{\boldsymbol{\theta}}^{*} & \text { Bootstrap parameter estimators. } \\ \widehat{m}^{*}, \widehat{\boldsymbol{m}}^{*} & \text { Bootstrap measurand estimator. } \\ \wp\left(\widehat{m}^{*}\right), \wp\left(\widehat{\boldsymbol{m}}^{*}\right) & \text { Bootstrap measurand distribution. } \\ \wp\left(\widehat{\boldsymbol{\theta}}^{*}\right) & \text { Bootstrap parameter distribution. } \\ h_{i}, w_{i} & \text { Vectors of weights used by the external and } \\ \xi(\varepsilon) & \text { the biased bootstraps. } \\ \gamma\left(w_{i}\right) & \text { Breakdown function. } \\ \Delta_{H}(\cdot) & \text { Data dispersion measure. } \\ B & \text { Hellinger's distance. } \\ B_{1}, B_{2} & \text { Number of simulations (iterations) for the } \\ \mathrm{BBQ} & \text { bootstrap. } \\ \mathrm{BBH} & \text { Number of simulations for the internal and the } \\ \mathrm{BOOT} & \text { external bootstraps. } \\ \mathrm{NLSCC} & \text { Biased bootstrap with quadratic norm func- } \\ \mathrm{Pdf} & \text { tion. } \\ & \text { Biased bootstrap with a Huber-like norm } \\ \text { function. } & \text { Bootstrap procedure. } \\ & \text { Nonlinear least squares estimation. } \\ & \text { Probability density function. } \\ & \text { Remote field eddy current. } \\ & \end{array}$

\section{INTRODUCTION}

I N MANY industrial applications, direct access to a measurand $(\boldsymbol{m})$ is not possible. Yet, as an estimation of the measurand is needed, the process must be treated as an inverse problem [1]. The characterization of the whole statistical knowledge about this quantity of interest $\boldsymbol{m}$ (quantity to be measured) is naturally given by the pdf $\wp(\boldsymbol{m})$. Most of the time, the largescale repetition of an experiment is not economically feasible. Therefore, Monte Carlo methods cannot be used. Bootstrap methods have proven to be a potentially useful alternative in accessing $\wp(\boldsymbol{m})$. Moreover, it is well known in practice that every observation in a data set does not play the same role in determining estimates, tests, or other statistics. This is due, for example, to a certain failure in the data acquisition or transmission processes. Such data are called outliers. When outliers are present in the data, a robust nonlinear regression strategy called biased bootstrap [2], [3] could be used for parameter and measurement estimation. The aim of this paper is then to appraise the measurand pdf using bootstrap techniques 
and to compare the results between different possible bootstrap schemes that have been implemented in [4] and [5]. In such a problem, difficulties are typically encountered when the size of the random sample is too small, and so asymptotic methods do not apply, or, more generally, when the distribution of the statistic of interest cannot be analytically expressed.

Bootstrap techniques were introduced by Efron [6] and have been mainly developed for the estimation of confidence intervals where few data are available [7]-[9]. Zoubir has used bootstrap techniques in a wide range of signal processing applications and he shows their potentially usefulness in [10]. The problem of uncertainty characterization of a measurand has been treated in a nonlinear Gaussian framework in [11] and [12]. However, multidimensional estimation problems are very difficult to deal with, since increasingly complex differentials and integrals have to be solved numerically. Furthermore, when the error distribution is unknown (i.e., noise) in a given application, it is still more difficult to solve the estimation problem. This complex problem of measurand pdf estimation with such more realistic assumptions has been approached in papers [4], [5], [13], obtaining promising results. In the case of the paper by Brahim-Belhouari et al. [13], the error limit bounds must be known, and the proposed method is deterministic.

The measurand uncertainty characterization using bootstrap techniques can be generally driven by the pdf estimation (stochastic method) in a nonlinear framework with unknown noise distribution (knowledge of limit bounds is not necessary) and with limited observed data. Section II presents the general formulation of the problem of measurand estimation. The biased bootstrap procedure for robust parameter estimation and its extension for measurand estimation are described in Section III, jointly with classical nonparametric bootstrap techniques. We also show how to improve uncertainty characterization when using the latter technique. A complex measurand-estimation problem of groove dimensioning using RFEC inspection is described in Section IV. Finally, some concluding remarks are given in Section V.

\section{Problem Statement}

In many applications, unknown quantities $m$ have to be estimated from a vector of observed values $\boldsymbol{y}$ (e.g., the direct observation of a quantity of interest is practically impossible). This may be encountered in several domains such as nondestructive testing or so-called indirect measurement. It is due to the impossibility of using transducers to measure $\boldsymbol{m}$ directly for any reason of harsh environment, long distance, etc. A measurand $\boldsymbol{m}$ can be defined as the best way to take advantage of the information given by the observed data $\boldsymbol{y}$. The first step of a measurand-estimation procedure consists in modeling the physical phenomenon under study. Therefore, building a model becomes a goal on its own. Indirect measurement systems can be formalized by two equations [1].

1) The observation equation is given by the classical nonlinear regression model:

$$
y_{i}=f\left(x_{i}, \boldsymbol{\theta}\right)+e_{i}, \quad i=1, \ldots, n
$$

where $\left\{x_{i}\right\}$ is an experimental design vector, assuming that $f(\cdot)$ is a known model structure with unknown parameter vector $\boldsymbol{\theta}$ (dimension $p$ ). Some fitting technique to estimate $\boldsymbol{\theta}$ can be used, e.g., nonlinear least squares, maximum likelihood, $\mathrm{M}$ estimator [14], etc.

2) The measurement equation (which is a nonlinear function of $\boldsymbol{\theta}$ )

$$
m_{\ell}=g_{\ell}(\boldsymbol{\theta}), \quad \ell=1, \ldots, r, \quad \text { with } \quad \boldsymbol{m}=\left\{m_{\ell}\right\}_{\ell=1}^{r} .
$$

The measurand is usually defined by a functional of the parametric model $\boldsymbol{m}=\mathcal{G}(f)$ (i.e., derivation, integration, interpolation, extrapolation, etc.). This relation is then transformed into a function of the parameters $\boldsymbol{\theta}$ such as in (2). It is supposed that the measurand(s) depends on at least one of the parameters $\left(\forall \ell, \exists k\right.$ such that $\left.\partial g_{\ell} / \partial \theta_{k} \neq 0\right)$. The final goal is therefore to obtain an accurate statistical characterization of the quantity(ies) of interest $\boldsymbol{m}$ (measurands).

\section{Bootstrap IN NONLINEAR REgRESSION AND MEASURAND ESTIMATION}

The first goal is then to determine the sampling distribution $\widehat{\wp} \widehat{\boldsymbol{\theta}}$ of $\widehat{\boldsymbol{\theta}}$. Efron [6], Freedman [15], Hinkley [9], and $\mathrm{Wu}$ [16] introduce and discuss many of the properties of the bootstrap method in classical regression analysis. When only reduced assumptions are made concerning the pdf of the errors $e_{i}$ (for example, assumptions could be that the error random variables belong to an independent identically distributed (i.i.d.) sequence of unknown law), bootstrap techniques can help to approximate the error pdf. These techniques are based on resampling a vector of a few observed residuals (see Section III-B), then a Monte Carlo procedure gives an estimation of the parameter pdf, and finally the pdf of the measurand can be approximated by its empirical equivalent. The first step for estimating the empirical density of the errors $\widehat{\wp}_{e}(\cdot)$ is working with residuals. The residuals are computed for all observed data $y_{i}$ using the proposed model $f(\cdot)$. Then, a fitting technique is used to obtain an estimation of $\boldsymbol{\theta}$. Since the real density $\wp_{e}(\cdot)$ is unknown, it is recommended to use a robust parameter and measurand estimation. Robust nonlinear regression deals with outlier accommodation. In order to accommodate points with large residuals, a biased bootstrap [3] technique that assures robustness has been used.

\section{A. Biased Bootstrap Parameter Estimator}

A biased bootstrap empirical method proposed in [3] is used for robust parameter estimation. Such a method identifies and downweights those data values that exert undue influence on a statistical estimator. The selected weights arise as resampling probabilities in a version of the weighted bootstrap and lead to a biased version of the uniform bootstrap. This approach does not need density estimation or the specification of a parametric family of distributions. The biased bootstrap requires two inputs: a distance measure between the uniform and the biased bootstrap distributions and some constraints. The distance measure used 
(e.g., power divergence), is described in [3] and is given by $D_{\rho}=\xi$, where $\xi$ is given by the following breakdown function:

$$
\xi(\varepsilon)= \begin{cases}{[\rho(1-\rho)]^{-1}\left\{1-(1-\varepsilon)^{1-\rho}\right\},} & \text { if } \rho \neq 1 \\ -\log (1-\varepsilon), & \text { if } \rho=1\end{cases}
$$

where $\rho$ is the exponent of the power divergence, and $\varepsilon$ is the initial breakdown point. It seems reasonable to choose an initial breakdown point $\varepsilon$ in the range $0<\varepsilon<1 / 2$. In practice, Hall and Presnell [3] suggest taking $\varepsilon \in(0.01,0.15)$, and $\rho$ varies in the range $0<\rho \leq 1$, including the Hellinger and Kullback-Leibler distances. The constraints are given by the measures of location and dispersion. The multivariate location estimation for a given bivariate sample $\left\{X_{i}, Y_{i}\right\}$ is obtained using

$$
\widehat{\boldsymbol{\theta}}=\arg \min _{\theta} \sum_{i=1}^{n} w_{i} \varphi\left(e_{i}\right)
$$

where the $w_{i}$ are positive weights and $\varphi\left(e_{i}\right)$ can be the quadratic norm function (BBQ)

$$
\varphi_{1}\left(e_{i}\right)=\left(y_{i}-f\left(x_{i}, \boldsymbol{\theta}\right)\right)^{2}
$$

or, to assure completely the robustness, the Huber-like norm can be used, as described in [12], and is given by the following equation $(\mathrm{BBH})$

$$
\varphi_{2}\left(e_{i}\right)=\frac{\Delta^{2}}{2}\left(\sqrt{1+\frac{4 \varphi_{1}\left(e_{i}\right)}{\Delta^{2}}}-1\right)
$$

where $\Delta>0$ and is a constant value. The measure of dispersion is given by

$$
\gamma\left(w_{i}\right)=\inf _{\theta} \sum_{i=1}^{n} w_{i} \varphi\left(e_{i}\right)
$$

The usual empirical distribution is given by the uniform bootstrap and considering that all weights have the same mass in all data points $\boldsymbol{w}_{\text {unif }}=(1 / n, \ldots, 1 / n)$. The initial values estimated for location and dispersion are given as follows: $\widehat{\boldsymbol{\theta}}=\widehat{\boldsymbol{\theta}}\left(\boldsymbol{w}_{\text {unif }}\right)$ and $\widehat{\gamma}=\widehat{\gamma}\left(\boldsymbol{w}_{\text {unif }}\right)$. Then, the level of dispersion is calibrated (e.g., minimized) by the biased weights (e.g., downweights) $w_{i}$. The biased weight function proposed in [3] is given in the following general form:

$$
w_{i}=\frac{1}{n} h_{[\xi]}\left(X_{i}, Y_{i} \mid \widehat{\boldsymbol{\theta}}, \widehat{\tau}, \widehat{\lambda}\right)
$$

where $h_{[\xi]}(\cdot)$ depends of the breakdown function $\xi$, that is

$h_{[\xi]}(\cdot)= \begin{cases}\left(1+(\rho-1)\left[\rho \xi-\widehat{\lambda}\left\{\varphi\left(e_{i}\right)-\widehat{\tau}\right\}\right]\right)^{1 /(\rho-1)}, & \text { if } \rho \neq 1 \\ \exp \left(\xi-\widehat{\lambda}\left\{\varphi\left(e_{i}\right)-\widehat{\tau}\right\}\right), & \text { if } \rho=1 .\end{cases}$

The values given by $(\widehat{\boldsymbol{\theta}}, \widehat{\tau}, \widehat{\lambda})$ satisfy the following equations:

1) criterion minimization $(\partial / \partial \boldsymbol{\theta})\left(\sum_{i=1}^{n} w_{i} \varphi\left(\widehat{e}_{i}\right)\right)=0$;

2) calculation of $\widehat{\tau}=\sum_{i=1}^{n} w_{i} \varphi\left(\widehat{e}_{i}\right)$, where $0<\widehat{\tau} \leq \widehat{\gamma}$;

3) $\widehat{\lambda}$ chosen by the bisection method given that $\sum_{i=1}^{n} w_{i}=1$.
If the distribution of $e_{i}$ is symmetric and unimodal, the true values of $\boldsymbol{\theta}$ are not changed by trimming. In the asymmetric case, they are altered, although they remain well defined as solutions of $\boldsymbol{\theta} \in \boldsymbol{\Theta} \subset \mathbb{R}^{p}$.

\section{B. Parameter pdf Estimation}

The conventional uniform bootstrap methods can be used to approximate the distribution of the biased estimator. The simple residuals $\widehat{e}_{i}$ are therefore obtained by using the following expression:

$$
\widehat{e}_{i}=y_{i}-f\left(x_{i}, \widehat{\boldsymbol{\theta}}\right) \text {. }
$$

The sample probability distribution of residuals $\widehat{\wp}_{e}$, is then approximated by a punctual statistical mass of $1 / n$ for each realization of $\widehat{e}_{i}$.

The next step is to draw the bootstrap samples (resampling with replacement) $e_{i}^{*}$ and $y_{i}^{*}$, given $\widehat{e}_{i} \sim \widehat{\wp}_{e}$ and $\widehat{\boldsymbol{\theta}}$ :

$$
y_{i}^{*}=f\left(x_{i}, \widehat{\boldsymbol{\theta}}\right)+e_{i}^{*}, \quad e_{i}^{*} \sim \widehat{\wp}_{e} .
$$

Indeed, $\widehat{\boldsymbol{\theta}}$ will be assumed to be the true parameters. Each realization of $\widehat{e}_{i}^{*}$ yields an estimation of $\widehat{\boldsymbol{\theta}}^{*}$ by the same minimization process that gave $\widehat{\boldsymbol{\theta}}$, e.g., (4).

Repeating $B$ independent bootstrap replications for $\widehat{\boldsymbol{\theta}}^{*}$ will give a random sample $\widehat{\boldsymbol{\theta}}^{* 1}, \ldots, \widehat{\boldsymbol{\theta}}^{* B}$, which can be used to estimate the bootstrap distribution of $\widehat{\boldsymbol{\theta}}^{*}$, and then the pdf of $\widehat{\boldsymbol{\theta}}$ could be approximated by $\wp\left(\widehat{\boldsymbol{\theta}}^{*}\right)$.

The bootstrap performance and approximation have been considered in both cases of Gaussian and non-Gaussian "assumptions" by working with residuals. The level of approximation can be improved by incorporating different priors to the residuals vector, while maintaining symmetric assumptions, such as the following.

1) Centered residuals: If one of the components of $\boldsymbol{\theta}$ is a translation parameter for the function $f(\cdot)$, then $\widehat{\wp}_{e}$ has zero mean. If not, $\widehat{\wp}_{e}$ may still be modified by translation to achieve zero mean [6], [15]

$$
\begin{aligned}
\widehat{r}_{i} & =y_{i}-f\left(x_{i}, \widehat{\boldsymbol{\theta}}\right) \\
\bar{r} & =\frac{1}{n} \sum_{i=1}^{n} \widehat{r}_{i} \\
\widehat{e}_{i} & =\widehat{r}_{i}-\bar{r} .
\end{aligned}
$$

2) Modified residuals: Bootstrapping modified residuals gives a smoothed (or weighted) version of residuals that can lead to a consistent estimator (e.g., weighted bootstrap) [17]

$$
\widehat{e}_{i}=\frac{\sqrt{\frac{n}{q}}\left(\widehat{r}_{i}-\bar{r}\right)}{\sqrt{1-\frac{p}{n}}} .
$$

The procedure of bootstrapping centered residuals can be modified. In linear regression, the bootstrap sample size 


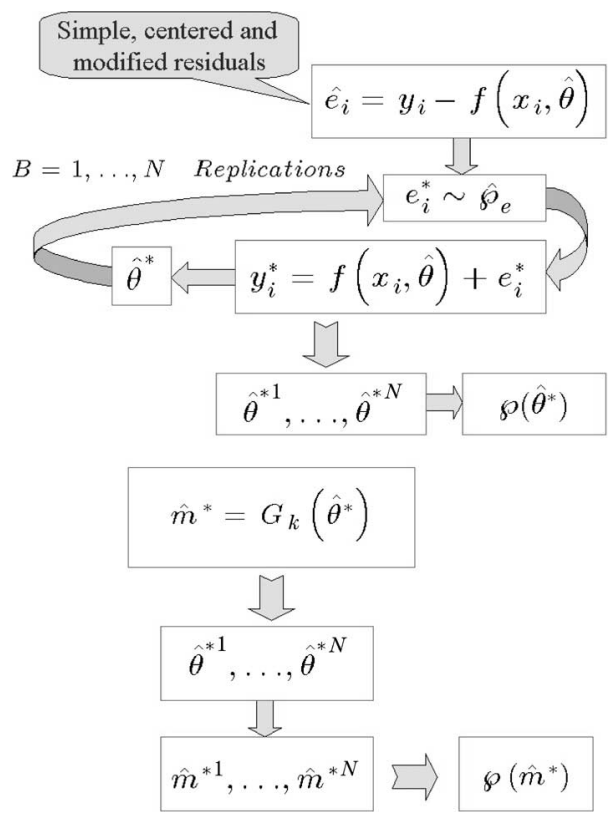

Fig. 1. Schematic illustration for parameter and measurand bootstrapping.

of $\widehat{e}_{i}^{*}$ is modified $(i=1, \ldots, q)$ so that $q<n$. However, in nonlinear regression, the $q$ factor value is modified so that $q<n$ and satisfying $q / n \rightarrow 0$, but the bootstrap sample size remains fixed $(i=1, \ldots, n)$. One restriction on $q$ is that $p / q$ should be reasonably small. For practical uses, $q$ needs to be specified for a fixed $n$, and its optimal choice depends on the parameter vector size. For example, one could begin with $q=n$ and then decrease this value until $q / n=0.5$ if $n$ is small and $q / n \rightarrow 0$ if $n \rightarrow \infty$.

\section{Measurand pdf Estimation}

An extended measurand vector is given by $\boldsymbol{m}=$ $\left[\theta_{1} \cdots m \cdots \theta_{p}\right]^{\mathrm{T}}$. It gives the nonlinear mapping $\boldsymbol{m}=G_{k}(\boldsymbol{\theta})$, where $k$ indicates the actual position of $m$ in $\boldsymbol{m}$. Once the parameter estimate $\widehat{\boldsymbol{\theta}}^{*}$ is computed, the measurand induced by the mapping $G_{k}(\cdot)$ is approximated according to the following equation:

$$
\widehat{\boldsymbol{m}}^{*}=g\left(\widehat{\boldsymbol{\theta}}^{*}\right)
$$

The measurand pdf $\wp_{m}(\boldsymbol{m})$ is then approximated by the bootstrap measurand pdf $\wp_{m}\left(\widehat{\boldsymbol{m}}^{*}\right)$, which is induced by $\widehat{\boldsymbol{m}}^{* 1}, \ldots, \widehat{\boldsymbol{m}}^{* B}$ using the different bootstrap replications $\widehat{\boldsymbol{\theta}}^{* 1}, \ldots, \widehat{\boldsymbol{\theta}}^{* B}$ in the nonlinear mapping $G_{k}(\cdot)$. The marginal pdf of the measurands of interest can be obtained after

$$
\wp\left(\widehat{m}_{\ell}^{*}\right)=\wp\left(g_{\ell}\left(\widehat{\boldsymbol{\theta}}^{*}\right)\right)=\int_{\mathbb{M}} \wp\left(\widehat{\boldsymbol{m}}^{*}\right) d \widehat{\boldsymbol{m}}_{-\ell}^{*}
$$

where $d \widehat{\boldsymbol{m}}_{-\ell}^{*}=d \widehat{m}_{1}^{*} \cdots d \widehat{m}_{\ell-1}^{*} d \widehat{m}_{\ell+1}^{*} \cdots d \widehat{m}_{r}^{*}$. A schema for parameter and measurand bootstrapping is shown in Fig. 1, where the procedure is iterated $B$ times to obtain the empirical pdf approximates.

\section{Nested Bootstrap}

In the bootstrap literature, one usually reads that the number of bootstrap simulation replications $B$ is approximately the same value as the sample size $n$. In parametric models with sufficient regularity, the bootstrap will yield an approximation of asymptotical statistics that is closer to the true distribution, at least in terms of orders of probability. By nesting the original bootstrap within another bootstrap, the approximation error can be reduced. In other words, the nested bootstrap (e.g., simulated bootstrap) can be used to improve the bootstrap convergence and to reduce the time of computations $(B \rightarrow \infty)$.

In order to diminish the stochastic error introduced by the bootstrap, $B$ must at least be $B \geq n^{2}$. In a simulated nested bootstrap, the number of replications in the inner bootstrap require $B_{1} \geq n^{2}$, and the number of replications in the outer bootstrap must satisfy $B_{2} \geq n^{3}$. Thus, the total number of replications must be $n^{5}$ to assure that the simulation stochastic error is not too large. This computational requirement can be prohibitive even in simple models with moderate sample sizes. A control variate approach is implemented to improve the bootstrap pdf convergence and approximation. This approach uses a leading term in the asymptotic expansion for the statistic of interest and its expectation to form a function that has the same expectation but smaller variance than the original statistic. The number of simulations required in the inner loop of the nested bootstrap is reduced to $B_{1} \geq n$, and to $B_{2} \geq n^{2}$ for the outer loop. Thus, the total number of replications required is reduced to $n^{3}$. Although the computational requirements involved in the latter modification can be substantial, the approach proposed gives a good improvement over the requirements needed for the standard nested bootstrap. A first approach was introduced in [18] for semiparametric models, such as nonlinear regression. This approach seems to give good results when the underlying distribution of the variables of interest is parametrically specified. Its extension to the estimation of the measurand $\boldsymbol{m}$ has been considered in [5].

Some restrictions are given for the assumptions concerning the error pdf: one needs independence of $x_{i}$, zero mean (symmetry), and uniform variance. When drawing replications from its empirical distribution $e_{i}^{(j)} \sim \widehat{\wp}_{e}$, there are complications to find $F_{\omega}(\boldsymbol{\beta}, t)$ (see the Appendix), but it should be possible to work out of its expectation function by giving a number of additional Monte Carlo replications. More details are given in the Appendix, where we also extend the application of [18, Th. 2] to the measurement estimation, since it is also considered as a semiparametric function.

\section{BOOTSTRAP ESTIMATION WITH A MEASUREMENT APPLICATION}

The measurand-estimation problem presented in [13] and [19] is considered here, as an example of a real problem. The RFEC inspection technique is used for dimensioning grooves that may occur in ferromagnetic conductive pipes. This task involves the depth and the length estimation of corrosion grooves, from observations of a pick-up coil signal phase $y$ at different positions $\boldsymbol{x}$ closest to the defect. A finite-element 


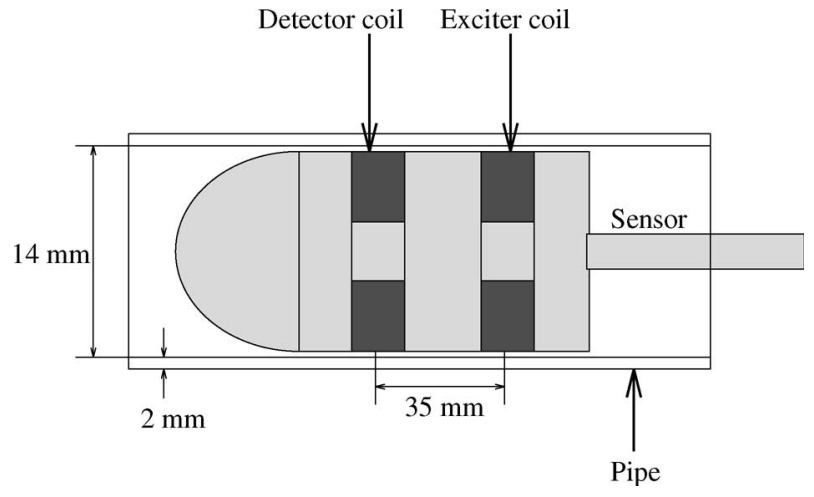

Fig. 2. Schematic illustration of the sensor pipe in the (RFEC) inspection problem.

calculation was performed in [19] to obtain a parametric model of the physical phenomenon. The dimensions of the groove to be estimated are linked to the parametric model through a polynomial function [see (16)]. The prior knowledge of the relationship between the groove parameters (length and depth) and the observed data (detector phase) is needed. We propose here the use of bootstrap techniques to obtain the statistical information about parameters and measurand estimates with the aim to qualify the performance of the different proposed estimators. Additionally, such techniques can be used to implement a scheme of model selection (this is not the aim in this paper, see [12] and [13]). In both cases, we use the modeling error (e.g., errors between the observed data and the nonlinear model) whose distribution is unknown.

Fig. 2 illustrates the typical experimental apparatus used for groove dimensioning. The sensor is pushed inside the pipe and, along with the coil position $\boldsymbol{x}$, the phase of the detector voltage $\boldsymbol{y}$ is acquired. The distance $(2 L)$ between the exciter and the detector coils is chosen so that the far-field condition is satisfied.

Taking into account the symmetry and the range of the data, several mathematical functions (e.g., nonlinear models) have been considered in [13] and [19] to approximate $\boldsymbol{y}$. The nonlinear model structure retained in [13] $\left(f_{2}(p=3)\right)$ as the best model is considered here

$$
\begin{aligned}
f(\boldsymbol{x}, \boldsymbol{\theta})=\theta_{1}( & \arctan \left(\theta_{2}\left(\boldsymbol{x}+\theta_{3}+L\right)\right) \\
& -\arctan \left(\theta_{2}\left(\boldsymbol{x}-\theta_{3}+L\right)\right) \\
& +\arctan \left(\theta_{2}\left(\boldsymbol{x}+\theta_{3}-L\right)\right) \\
& \left.-\arctan \left(\theta_{2}\left(\boldsymbol{x}-\theta_{3}-L\right)\right)\right)
\end{aligned}
$$

where $L$ is an experimental constant (half-distance between coils equal to $17.5 \mathrm{~mm}$ ). The length of vectors $\boldsymbol{x}$ and $\boldsymbol{y}$ is $n=118$. Fig. 3 shows an example of simulated observed data computed by a finite-element code for given groove parameters.

The measurement goal is to estimate the size of the defect [depth $(d)$ and length $(l)$ ] from the knowledge of detector phase. The measurement quantity can be expressed as a function of the optimal parameters of the model. An algebraic

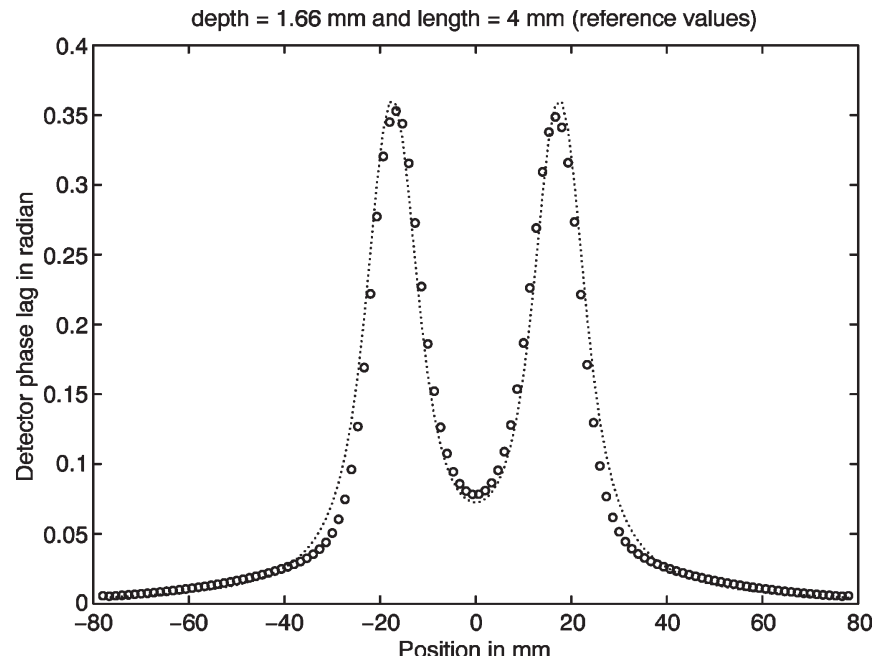

Fig. 3. Finite element simulated data $\boldsymbol{y}$ versus $\boldsymbol{x}$ (circles) and modeled data using the parameter mean value obtained with the bootstrap-BBH $(\cdots)$.

TABLE I

STATISTICS OBTAINED USING THE NLS ESTIMATOR AND BOOTSTRAP TECHNIQUES FOR $\boldsymbol{\theta}$ AND THE MEASURANDS $\boldsymbol{m}$

\begin{tabular}{|c||c|c|c|c|c|c|}
\hline Boot.-NLS $^{1}$ & True & Mean & Median & Bias & Var & std \\
\hline \hline$\theta_{1}$ & unknown & 0.3136 & 0.2959 & 0.0196 & 0.0064 & 0.0798 \\
\hline$\theta_{2}$ & unknown & 0.1833 & 0.1830 & $4.0 \mathrm{e}-4$ & $6.6357 \mathrm{e}-5$ & 0.0081 \\
\hline$\theta_{3}$ & unknown & 3.4981 & 3.5662 & 0.0903 & 0.3984 & 0.6312 \\
\hline$d$ & 1.6600 & 1.6894 & 1.6930 & 0.0294 & 0.0248 & 0.1575 \\
\hline$l$ & 4.0000 & 4.4600 & 4.5523 & 0.4600 & 1.1109 & 1.0540 \\
\hline Boot.-NLS & & & & & & \\
\hline \hline$\theta_{1}$ & unknown & 0.3095 & 0.2940 & 0.0155 & 0.0056 & 0.0746 \\
\hline$\theta_{2}$ & unknown & 0.1839 & 0.1838 & $2.0 \mathrm{e}-4$ & $5.1572 \mathrm{e}-5$ & 0.0072 \\
\hline$\theta_{3}$ & unknown & 3.5272 & 3.5899 & 0.0612 & 0.3472 & 0.5892 \\
\hline$d$ & 1.6600 & 1.6813 & 1.6826 & 0.0213 & 0.0202 & 0.1421 \\
\hline$l$ & 4.0000 & 4.5099 & 4.5977 & 0.5099 & 0.9986 & 0.9993 \\
\hline
\end{tabular}

form for $g$ is chosen; $g$ can be taken as a bilinear polynomial function of $\boldsymbol{\theta}$, which may be written as follows:

$$
\begin{gathered}
\widehat{d}=g_{d}(\boldsymbol{\theta})=\sum_{k, h} c_{k h}^{d} \theta_{k} \theta_{H}+\sum_{k} c_{k}^{d} \theta_{k} \\
\widehat{l}=g_{l}(\boldsymbol{\theta})=\sum_{k, h} c_{k h}^{l} \theta_{k} \theta_{H}+\sum_{k} c_{k}^{l} \theta_{k} .
\end{gathered}
$$

The coefficients $\left(c_{k h}^{d}, c_{k}^{d}\right)$ and $\left(c_{k h}^{l}, c_{k}^{l}\right)$ have been computed in the least squares sense [19] for several real defect dimensions. Thus, an analytical form for the measurement vector $\widehat{\boldsymbol{m}}=$ $\left[\widehat{d}, \widehat{l}^{\mathrm{T}}\right.$ as a function of $\boldsymbol{\theta}$ is obtained for the candidate model.

Table I shows the results obtained for the weighted bootstrap technique using the NLS estimator to obtain the $\widehat{d}$ and $\widehat{l}$ estimates. The empirical statistical information about these estimates is also obtained, and the mean value for both measurands is near the reference value. On the other hand, Table II presents some statistical results obtained using the biased bootstrap estimators with BBQ and $\mathrm{BBH}$; especially in the case when BBH is used, one notes the minimum dispersion of all measurand populations. The values of reference of the 
TABLE II

STATISTICS OBTAINED By THE DIFFERENT BoOTSTRAP TECHNIQUES FOR $\boldsymbol{\theta}$ AND THE MEASURANDS $\boldsymbol{m}$

\begin{tabular}{|c||c|c|c|c|c|c|}
\hline Boot.-BBQ $^{1}$ & True & Mean & Median & Bias & Var & std \\
\hline \hline$\theta_{1}$ & unknown & 0.3163 & 0.2953 & 0.0223 & 0.0092 & 0.0958 \\
\hline$\theta_{2}$ & unknown & 0.1834 & 0.1832 & 0.0003 & $6.5982 \mathrm{e}-5$ & 0.0081 \\
\hline$\theta_{3}$ & unknown & 3.4947 & 3.5722 & 0.0937 & 0.4052 & 0.6365 \\
\hline$d$ & 1.6600 & 1.6891 & 1.6915 & 0.0291 & 0.0248 & 0.1573 \\
\hline$l$ & 4.0000 & 4.4516 & 4.5730 & 0.4516 & 1.1574 & 1.0758 \\
\hline Boot.-BBQ & & & & & & \\
\hline \hline$\theta_{1}$ & unknown & 0.3111 & 0.2943 & 0.0171 & 0.0065 & 0.0807 \\
\hline$\theta_{2}$ & unknown & 0.1841 & 0.1839 & 0.0004 & $6.7850 \mathrm{e}-5$ & 0.0082 \\
\hline$\theta_{3}$ & unknown & 3.5259 & 3.5857 & 0.0625 & 0.3988 & 0.6315 \\
\hline$d$ & 1.6600 & 1.6761 & 1.6826 & 0.0161 & 0.0257 & 0.1603 \\
\hline$l$ & 4.0000 & 4.5106 & 4.5901 & 0.5106 & 1.1414 & 1.0684 \\
\hline Boot.-BBH & & & & & & \\
\hline \hline$\theta_{1}$ & unknown & 0.3009 & 0.2908 & 0.0068 & 0.0027 & 0.0516 \\
\hline$\theta_{2}$ & unknown & 0.1841 & 0.1837 & $4.0 \mathrm{e}-4$ & $5.5635 \mathrm{e}-5$ & 0.0075 \\
\hline$\theta_{3}$ & unknown & 3.5957 & 3.6480 & 0.0078 & 0.2598 & 0.5097 \\
\hline$d$ & 1.6600 & 1.6713 & 1.6824 & 0.0113 & 0.0193 & 0.1390 \\
\hline$l$ & 4.0000 & 4.6173 & 4.6838 & 0.6173 & 0.7975 & 0.8930 \\
\hline & & & & & & \\
\hline
\end{tabular}

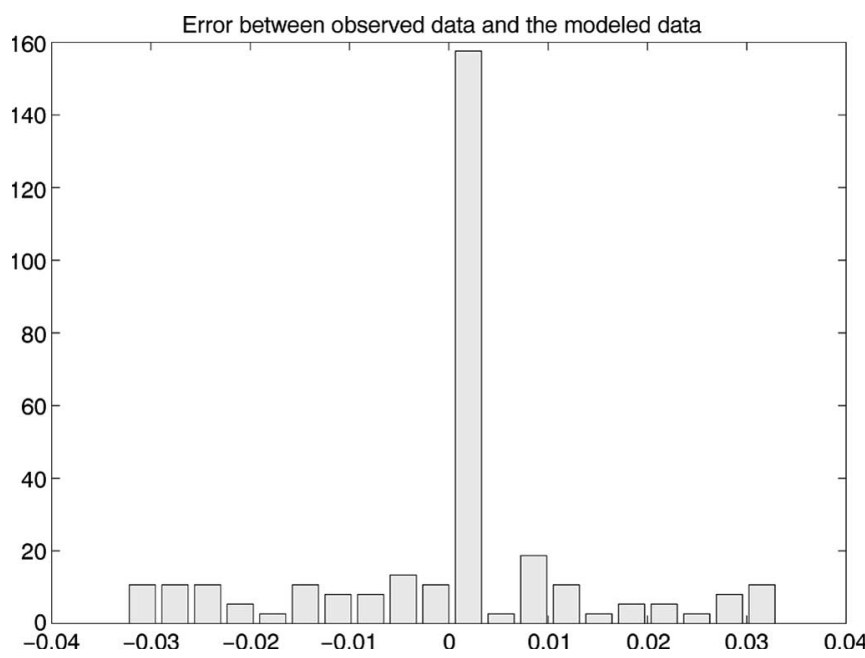

Fig. 4. Error vector obtained by $\boldsymbol{y}-f\left(\boldsymbol{x}, \widehat{\boldsymbol{\theta}}_{\mathrm{NLS}}\right)$.

defects are $d=1.66 \mathrm{~mm}$ and $l=4 \mathrm{~mm}$. It can be seen in Fig. 4 that the errors are centered. Of course, there is no evidence that the distribution is normal, because it is actually unknown (the residuals are obtained using the first parameter estimates into the proposed model and the data $y$ ).

Fig. 5 illustrates several replications of the modeled data given by the $\mathrm{BBH}$ estimator plus empirical resampled errors. The circles are the same data as presented in Fig. 3 and it may be seen that they are inside the statistical domain of the proposed model. In the case where a noncentered weighted bootstrap ${ }^{1}$ (Tables I and II) procedure is used for residuals (where the $\bar{r}$ element of (12) is removed), the statistical information obtained by the NLS and BBQ estimators is very similar (both estimators take into account that the error pdf is centered). Moreover, the weighted bootstrap (recentered ${ }^{2}$ Tables I and II) also gives similar results, but the statistics of the measurements ( $l$ and $d$ )

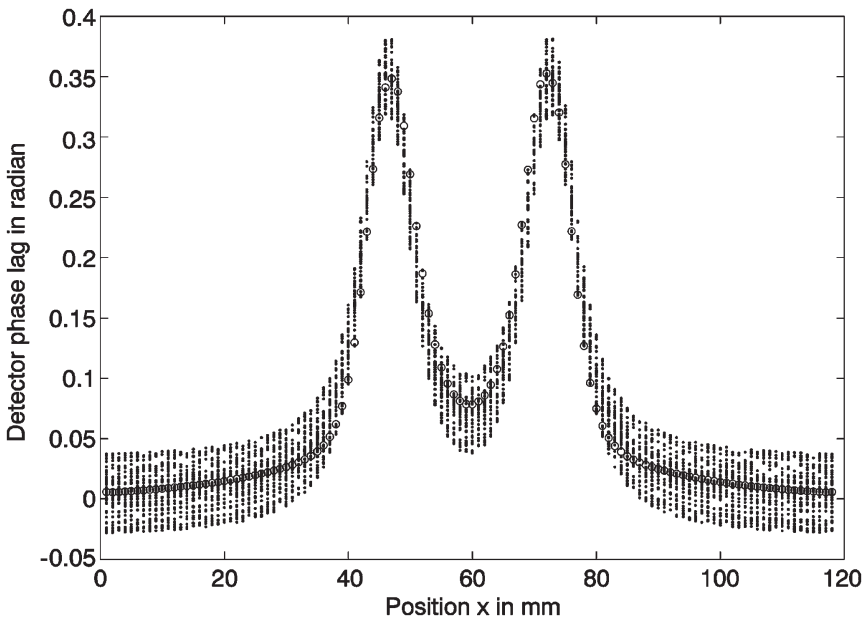

Fig. 5. Simulated data (circles) and some replications of modeled data plus noise resamples for the scheme $\mathrm{BBH}(\cdot)$.
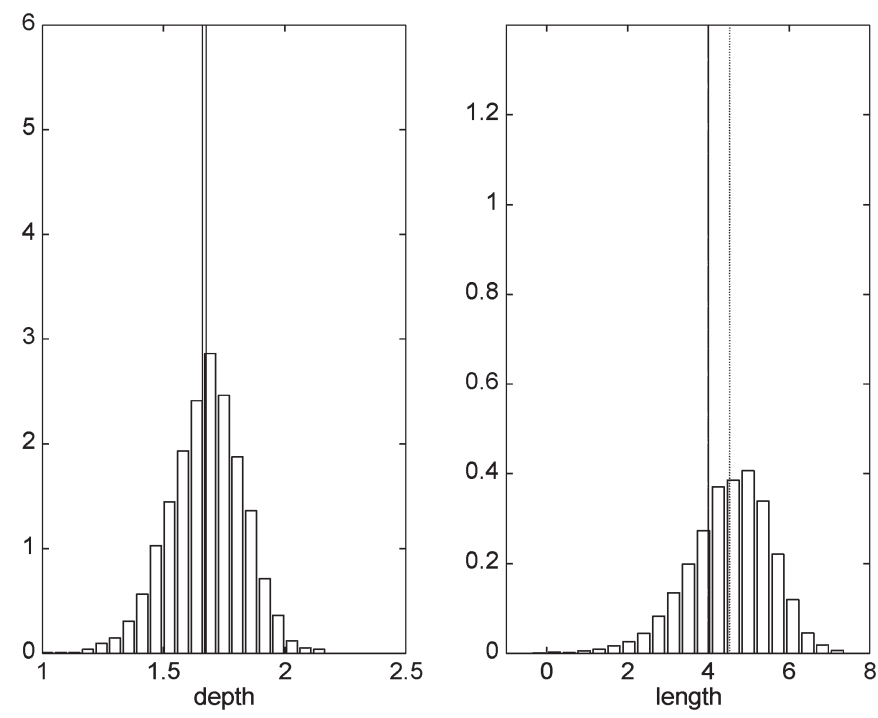

Fig. 6. Histograms obtained from measurand populations with bootstrap$\mathrm{NLS}^{2}$, reference values (-), and mean values $(\cdots)$. The axe $y$ indicates the nonnormalized empirical probability.

are more accurate. For example, the bias between the measurand reference values and the mean values is reduced and the recentering factor admits more robustness in the optimization procedure. The $\mathrm{BBH}$ estimator gives the most concentrated populations and, therefore, the statistics obtained have the smallest values in general. The correlation between histograms obtained from measurand populations with the bootstrap-NLS ${ }^{2}$ and the bootstrap-BBH can be done by comparing Figs. 6 and 8 , where the dotted lines represent the mean value and the solid lines represent the actual reference values. Finally, one can do the same comparison with the bootstrap-BBQ ${ }^{2}$ (see Fig. 7) and the bootstrap-BBH (see Fig. 8).

For these application data, we cannot notice the outliers since the data dispersion seems to be regular. Nevertheless, the robust estimation works well as it has been seen in results given when the $\mathrm{BBH}$ was used in [5] (working with synthetic data), and results presented in this paper, where the measurand dispersions are the smallest. On the other hand, the simulation time is a great issue; for example, for 5000 bootstrap iterations 

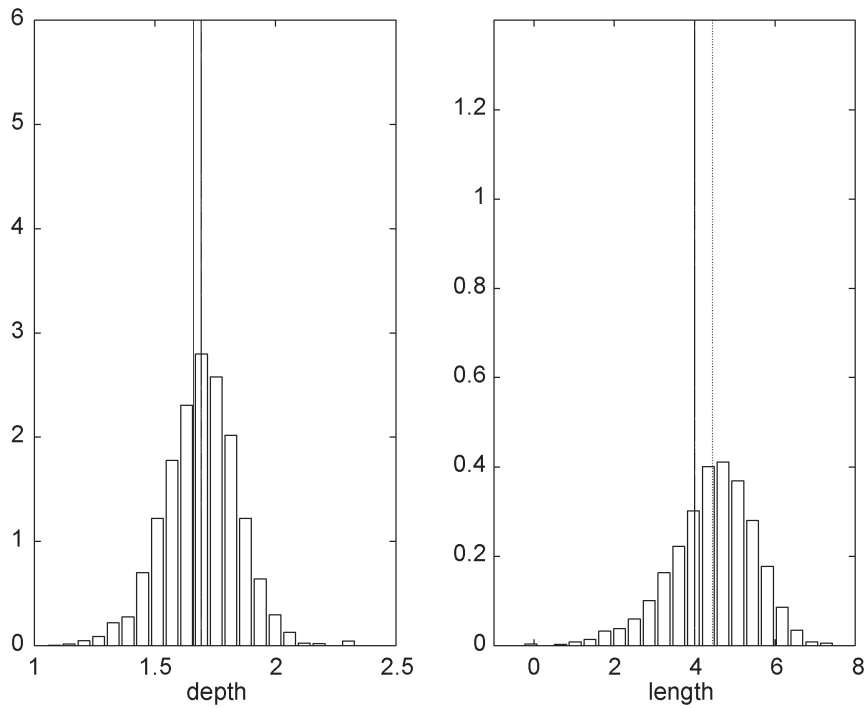

Fig. 7. Histograms obtained from measurands populations with bootstrap$\mathrm{BBQ}^{2}$, reference values $(-)$, and mean values $(\cdots)$. The axe $y$ indicates the nonnormalized empirical probability.
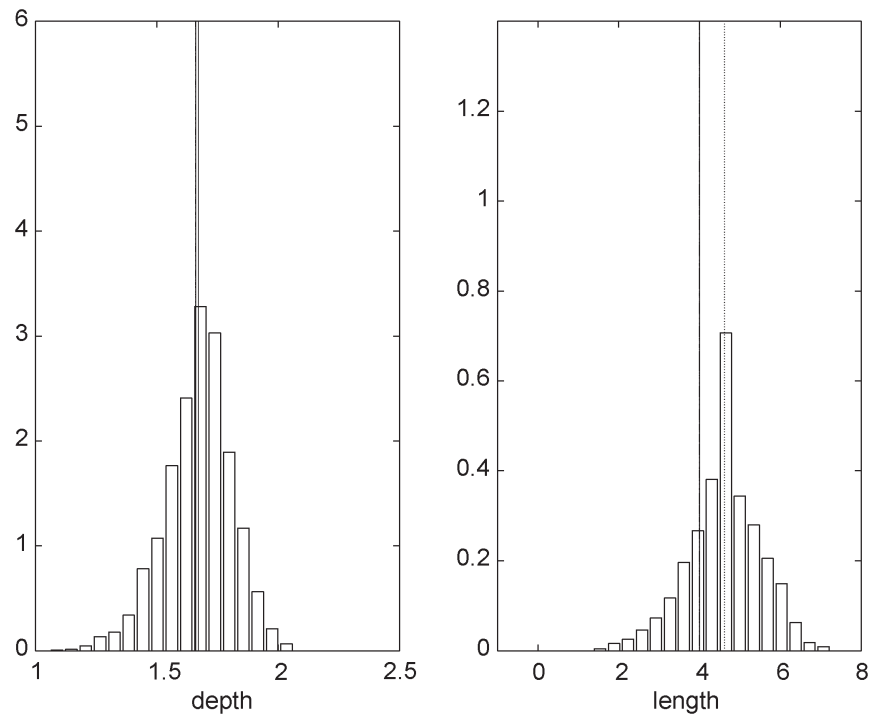

Fig. 8. Histograms obtained from measurand populations with bootstrap$\mathrm{BBH}$, reference values (-), and mean values $(\cdots)$. The axe $y$ indicates the nonnormalized empirical probability.

of the BBH scheme for the proposed model used for RFEC application, the time needed to achieve convergence of simulation was $58.21 \mathrm{~min}$, whereas NLS or BBQ simulation time was in the interval of 12.07-6.67 min when using an ultra Sparc 5 system (256-MB RAM) and MATLAB version 5.2. In fact, the level of approximation not only depends on the model $f(\cdot)$, but also on the polynomial (nonlinear) measurand mapping $g(\cdot)$.

\section{CONCLusion}

The bootstrap measurand estimation offers good approximation results that are near the measurands of reference. In particular, the level of accuracy depends on the measurement mapping. Moreover, this level could be improved using prior information on the residuals in a correct way. Under Gaussian and unknown noise assumptions (symmetric or nonsymmetric), bootstrap gives a good approximation of the measurand pdf. It works very well in the case of robust estimation under an unknown error distribution. Bootstrap converges with a small number of iterations (even if the time of convergence depends on the optimization problem). The nested bootstrap is used successfully to significantly reduce the number of iterations, and the variance is accurately approximated [5]. The only restrictions lie in the noise pdf symmetry assumptions. It has been shown that the nested and weighted bootstraps give competitive results. As presented in [19], the model used here is best adapted for small defects. Modeling noise is then reduced, thus improving the robustness of the model and the accuracy of the approximation. A bootstrap selection procedure is not of importance here, since several procedures were suggested in [12] and [13]. However, if a selection procedure is required to compare the suggested ones, the procedure given in [17] could be applied and extended to the measurand-estimation problem.

\section{APPENDIX}

Let $\boldsymbol{\beta}=[\boldsymbol{\theta}, \boldsymbol{m}]$ be an extended parameter vector. The distribution function of any statistic $T(\boldsymbol{\beta})$ of $\boldsymbol{\beta}$ is given by $\Gamma(\boldsymbol{\beta}, T(\boldsymbol{\beta}))=E_{\beta}\{I(T(\boldsymbol{\beta}) \leq t)\}$ [18], and the function of interest is $H(T(\boldsymbol{\beta}), t)=I(T(\boldsymbol{\beta}))$. It is necessary to assume that $T(\boldsymbol{\beta})$ has a limiting behavior with finite sample knowledge of the leading term, of which an Edgeworth expansion is given as follows:

$$
T(\boldsymbol{\beta})=\omega\left(e_{1}, e_{2}, \ldots, e_{n} ; \boldsymbol{\beta}\right)+O_{p}\left(n^{-q / 2}\right)
$$

where $\omega(\boldsymbol{\beta})=\omega\left(e_{1}, e_{2}, \ldots, e_{n} ; \boldsymbol{\beta}\right)$ is an approximated statistic of $T(\boldsymbol{\beta})$, given the error random sample $e_{i} \sim \widehat{\wp}_{e}(\boldsymbol{\theta})$ from its known parameterized density, and $F_{\omega}(\boldsymbol{\beta}, t)=E_{\beta}\{I(\omega(\boldsymbol{\beta}) \leq$ t) \} known up to the parameters. The statistic $F_{\omega}(\boldsymbol{\beta}, t)$ is pivotal and does not depend on $\boldsymbol{\beta}$ when $q=1, F_{\omega}(\boldsymbol{\beta}, t)=F_{\omega}(t)$. The knowledge of $T$ and $H(T(\boldsymbol{\beta}), t)$ is used to form a control variate that eliminates the leading stochastic term in $H(T(\boldsymbol{\beta}), t)$ and then simulate the remainder.

Given $\boldsymbol{\beta}$ and knowledge of $\omega(\boldsymbol{\beta})$, one generates $e_{i}^{(j)} \sim$ $\widehat{\wp}_{e}(\boldsymbol{\theta}), \quad j=1, \ldots, B_{1}$, forming $T^{(j)}(\boldsymbol{\beta})$ and $\omega^{(j)}(\boldsymbol{\beta})=$ $\omega\left(e_{i}^{(j)} ; \boldsymbol{\beta}\right)$, and defining

$\widetilde{H}\left(T^{(j)}(\boldsymbol{\beta}), \omega^{(j)}(\boldsymbol{\beta}), t\right)=I\left(T^{(j)}(\boldsymbol{\beta})\right)-I\left(\omega^{(j)}(\boldsymbol{\beta})\right)+F_{\omega}($

and the average value over the Monte Carlo is

$$
\begin{aligned}
& \widetilde{\Gamma}_{B_{1}}(\widehat{\boldsymbol{\beta}}, t)=\frac{1}{B_{1}} \sum_{j=1}^{B_{1}} \widetilde{H}\left(T^{(j)}(\widehat{\boldsymbol{\beta}}), \omega^{(j)}(\widehat{\boldsymbol{\beta}}), t\right) \\
& \widetilde{\Gamma}_{B_{1}}(\widehat{\boldsymbol{\beta}}, t)=\Gamma\left(\boldsymbol{\beta}_{0}, t\right)+O_{p}\left(n^{-1(k+1) / 2}\right)+O_{p}\left(n^{-q / 2} B_{1}^{-1 / 2}\right)
\end{aligned}
$$

It is required that $B_{1} \geq n^{k+1-q}$ to ensure that the Monte Carlo error does not dominate. In the outer bootstrap, $B_{2}$ replications are necessary, and the function of interest is given by $\widetilde{T}_{1}(\boldsymbol{\beta})=$ $\widetilde{\Gamma}_{B_{1}}(\widehat{\boldsymbol{\beta}}, T(\boldsymbol{\beta}))$, where $\widetilde{T}_{1}(\boldsymbol{\beta})=\Gamma(\boldsymbol{\beta}, T(\boldsymbol{\beta}))+O_{p}\left(n^{-(k+1) / 2}\right)$, and the distribution function is given by $\widetilde{\Gamma}_{1}=$ $E_{\beta}\left\{I\left(\widetilde{T}_{1}(\boldsymbol{\beta}) \leq t_{1}\right)\right\}$. 
Applying the previous ideas, one defines

$\widetilde{H}_{1}\left(\widetilde{T}_{1}^{(l)}(\boldsymbol{\beta}), \omega^{(l)}(\boldsymbol{\beta}), t_{1}\right)=I\left(\widetilde{T}_{1}^{(l)}(\boldsymbol{\beta})\right)-I\left(F_{\omega}\left(\omega^{(l)}(\boldsymbol{\beta})\right)\right)+t_{1}$

and its average over the Monte Carlo is

$\widetilde{\Gamma}_{1 B_{2}}(\widehat{\boldsymbol{\beta}}, t)=\frac{1}{B_{2}} \sum_{l=1}^{B_{2}} \widetilde{H}_{1}\left(T_{1}^{(l)}(\widehat{\boldsymbol{\beta}}), \omega^{(l)}(\widehat{\boldsymbol{\beta}}), t_{1}\right)$

$\widetilde{\Gamma}_{1 B_{2}}(\widehat{\boldsymbol{\beta}}, t)=\widetilde{\Gamma}_{1}\left(\boldsymbol{\beta}_{0}, t_{1}\right)+O_{p}\left(n^{-1(k+2) / 2}\right)+O_{p}\left(n^{-q / 2} B_{2}^{-1 / 2}\right)$.

In general, it is required that $B_{2} \geq n^{k+2-q}$, and then, for a common case $k=q=1, B_{1} \geq n$ and $B_{2} \geq n^{2}$, and the total replications are $n^{3}$.

\section{ACKNOWLEDGMENT}

The authors would like to thank M.-E. Davoust for allowing them to use her data. The authors would also like to thank J. Oksman, Y. Moudden, and S. Brahim-Belhouari for their fruitful comments about this paper. Moreover, they would like to acknowledge the diligent review and useful comments given by reviewers, which helped in improving this paper.

\section{REFERENCES}

[1] G. Fleury, "Optimal nonlinear modeling and reparametrization," in Proc. IEEE Int. Workshop Intell. Signal, Sep. 1999, pp. 72-76.

[2] P. Hall and B. Presnell, "Applications of intentionally biased Bootstrap methods," in Proc. Int. Congr. Math., Berlin, vol. III, Documenta Mathematica, Fak. Math., Biefield, Germany: Univ. Biefield, 1998, pp. 257-266. [Online]. Available: http://www.mathematik.uni-biefield. de/documenta/xvol-icm/12/12.html

[3] _ - "Intentionally biased Bootstrap methods," J. R. Stat. Soc., Ser. B, vol. 61 , no. 1, pp. 143-158, 1999.

[4] J. I. De la Rosa and G. Fleury, "Bootstrap density approximation for measurement purposes," in Proc. IASTED Conf. Signal Process. Commun., Marbella, Spain, Sep. 2000, pp. 357-361.

[5] — - "Bootstrap methods applied to indirect measurement," in Proc. Phys. Signal Image Process. Conf., Marseille, France, Jan. 2001, pp. 57-62.

[6] B. Efron, "Bootstrap methods : Another look at the Jackknife," Ann. Stat., vol. 7, no. 1, pp. 1-26, Jan. 1979.

[7] N. DiCiccio and J. P. Romano, "A review of Bootstrap confidence intervals (with discussion)," J. R. Stat. Soc., Ser. B, vol. 50, no. 3, pp. 338-370, 1988.

[8] B. Efron, "Better Bootstrap confidence interval," J. Amer. Stat. Assoc., vol. 82, no. 397, pp. 171-185, Mar. 1987.

[9] D. V. Hinkley, "Bootstrap methods," J. R. Stat. Soc., Ser. B, vol. 50, no. 3, pp. 321-337, 1988.

[10] A. M. Zoubir, "The Bootstrap and its application in signal processing," IEEE Signal Process. Mag., vol. 15, no. 1, pp. 56-76, Jan. 1998.

[11] S. Brahim-Belhouari and G. Fleury, "Probability distribution in nonlinear estimation-A measurement dedicated approach," in Proc. 9th IEEE Workshop Stat. Signal Array, Portland, OR, 1998, pp. 395-398.
[12] S. Brahim-Belhouari, M. Kieffer, G. Fleury, L. Jaulin, and E. Walter, "Model selection via worst-case criterion for nonlinear bounded-error estimation," IEEE Trans. Instrum. Meas., vol. 49, no. 3, pp. 653-658, Jun. 2000.

[13] S. Brahim-Belhouari, G. Fleury, and M. E. Davoust, "Model selection based on robustness criterion with measurement application," in Proc. SPIE Conf. Math. Model. Bayesian Estim. Inverse Probl., Denver, CO, 1999, vol. 3816, pp. 196-202.

[14] B. T. Poljak and J. Z. Tsypkin, "Robust identification," Automatica, vol. 16 , no. 1, pp. 53-63, 1980.

[15] D. A. Freedman, "Bootstrapping regression models," Ann. Stat., vol. 9, no. 6, pp. 1218-1228, Nov. 1981.

[16] C. F. J. Wu, "Jackknife, Bootstrap and other resampling methods in regression analysis," Ann. Stat., vol. 14, no. 4, pp. 1261-1295, Dec. 1986.

[17] J. Shao, "Bootstrap model selection," J. Amer. Stat. Assoc., vol. 91, no. 434, pp. 655-665, Jun. 1996.

[18] B. W. Brown, 1996 "Simulation variance reduction for bootstrapping," NSF Bootstrap Symposium. [Online]. Available: //emlab.berkeley.edu/ nsf96wp.html

[19] M.-E. Davoust, G. Fleury, and J. Oksman, "A parametric estimation approach for grooves dimensioning using remote field eddy current inspection," Res. Nondestruct. Eval., vol. 11, pp. 39-57, 1999.

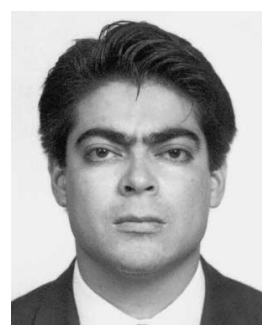

José I. De la Rosa (S'01-A'03-M'04) was born in Zacatecas, Mexico, on March 19, 1972. He received the B.Sc. degree in electronics and telecommunications engineering from the Universidad Autónoma de Zacatecas, in 1995, the M.Sc. degree in digital systems from the Instituto Politécnico Nacional (CITEDI), Mexico City, Mexico, in 1998 and the $\mathrm{Ph} . \mathrm{D}$. degree in signal processing and control from the Université de Paris-Sud, Orsay, France, in 2002.

He was a Ph.D. student within the Measurement Department (now Department of Signal Processing and Electronic Systems) at École Supérieure d'Électricité (SUPELEC) Gifsur-Yvette, France. He is presently an Associate Researcher at the Signal Processing Laboratory of the engineering faculty of the Universidad Autónoma de Zacatecas. His main research interests are statistical signal processing, modeling and system identification, and signal and image processing in robotics.

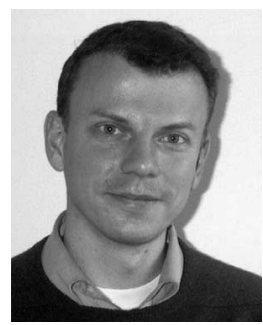

Gilles A. Fleury was born in Bordeaux, France, on January 8, 1968. He received the M.S. degree from the École Supérieure d'Électricité (SUPELEC), Gif-sur-Yvette, France, in 1990, the Ph.D. degree in signal processing from the Université de Paris-Sud, Orsay, France, in 1994, and the Habilitation a Diriger les Recherches (HDR) degree in 2003.

$\mathrm{He}$ is presently a Professor within the Department of Signal Processing and Electronic Systems of SUPELEC. He has worked in the areas of inverse problems and optimal design. His current research interests include bioinformatics, optimal nonlinear modeling, and nonuniform sampling. 